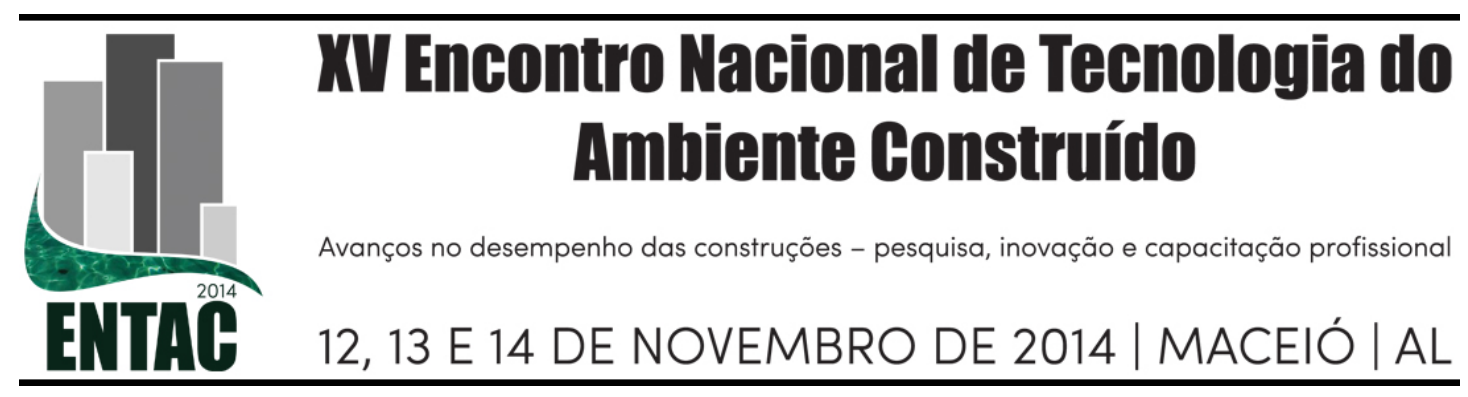

\title{
ESTUDO DA INFLUÊNCIA DO AGREGADO RECICLADO DE CONCRETO DE PRÉ-MOLDADOS NA RESISTÊNCIA DO CONCRETO
}

\author{
LEITE, Mônica B. (1); COSTA, Josué A. (2)
}

(1) PPGECEA/DTEC - Universidade Estadual Feira de Santana, +55 (75) 3161-8117, email: mleite.uefs@gmail.com (2) DTEC - Universidade Estadual Feira de Santana, email: josueandradecosta@gmail.com

\section{RESUMO}

A construção civil apresenta uma importante participação na economia brasileira, sendo responsável pelo consumo de grandes quantidades de matérias-primas, como também na geração de resíduos durante os processos construtivos. Os resíduos de concreto provenientes de centrais dosadoras, fábrica de pré-moldados, novas construções, demolições e etc, são significantes na composição total do Resíduo de Construção e Demolição-RCD, aproximadamente $29 \%$ juntamente com blocos, por isso, é interessante o aproveitamento destes resíduos. A utilização de resíduos de concreto provenientes de pré-moldados possibilita obter agregados reciclados com maior controle das propriedades do concreto reciclado produzido. Assim, este trabalho foi desenvolvido com objetivo de estudar a influência da utilização de agregados reciclados de concreto pré-moldado para a produção de novos concretos. Para o desenvolvimento do estudo foi realizada a caracterização dos agregados graúdos reciclados que foram obtidos a partir do beneficiamento de resíduos de concreto de uma fábrica de prémoldados. Foram produzidas duas misturas de concreto de referência, para alcançar as resistências de 25 e $45 \mathrm{MPa}$, aos 28 dias, cujas relações água/cimento foram de 0,69 e 0,40 , respectivamente. A partir dos concretos de referência foram produzidos concretos fazendo a substituição de $50 \%$ do agregado graúdo natural por agregado graúdo reciclado de concreto. A influência do agregado reciclado foi avaliada quanto a resistência à compressão e resistência à tração por compressão diametral dos concretos, aos 7 e 28 dias. Os resultados indicaram que os concretos produzidos com agregado graúdo reciclado de concreto pré-moldado apresentaram melhor desempenho mecânico que os concretos de referência.

Palavras-chave: Concreto reciclado; Propriedades mecânicas; Aproveitamento de resíduos.

\section{ABSTRACT}

The construction sector is very important to Brazilian economy and presents a great consumption of natural resources and waste generation. Concrete waste that comes from pre-mixing concrete industry, pre-cast industry, new construction sites, or demolition sites shows a great amount in the construction and demolition waste-CDW 
(around 29\% if it is added with concrete blocks). Recycling this material allows achieving good quality aggregates for new concrete production. Thus, this work aim is to study the influence of using recycled coarse concrete aggregate-RCCA from pre-cast industry to produce new concrete. Two families of concrete were produced to reach 25 and $45 \mathrm{MPa}$ compressive strength at 28-days. The water/cement ratios were 0,69 and 0,40 , respectively. The recycled concrete were produced using $50 \%$ of replacement of natural aggregate by RCCA using the same concrete mixtures (25 and 40MPa). Compressive and split tensile strengths were evaluated at 7 and 28-days-of-age. The results showed that recycled concrete produced with $R C C A$ from pre-cast industry has better mechanical performance than the concretes used as reference.

Keywords: Recycled concrete; Mechanical properties; Recycling.

\section{INTRODUÇÃ̃O}

A construção civil apresenta uma importante participação na economia brasileira, sendo responsável pelo consumo de grandes quantidades de matérias-primas, como também na geração de resíduos durante os processos construtivos. A Construção Civil é a responsável por gerar a maior parcela dos resíduos sólidos urbanos no Brasil. Um estudo realizado por Pinto (2005) mostra a composição dos resíduos sólidos urbanos, em onze municípios da região sudeste, e o predomínio do resíduo de construção e demolição sobre todos os outros resíduos urbanos. Aproximadamente $61 \%$ dos resíduos gerados nas cidades são compostos por RCD, $28 \%$ por resíduos domiciliares e $11 \%$ por outros resíduos. Em muitas cidades brasileiras, os resíduos da construção e demolição (RCD) são depositados em locais inadequados, como em vias públicas, lixões a céu aberto e área de aterro, agravando ainda mais os problemas ambientais existentes.

Os RCD são resíduos provenientes de novas construções que podem ser residenciais, comerciais e industriais, construções de infra-estrutura nas cidades, obras de reformas, ampliações e demolições. Segundo Pinto (2005), 59\% dos RCD são gerados por reformas, ampliações e demolições, $20 \%$ por novas construções e $21 \%$ por edificações novas acima de $300 \mathrm{~m}^{2}$.

O RCD é composto por vários materiais e cada região do Brasil pode apresentar uma composição diferente, dependendo do tipo de obra. Em geral o RCD é composto por: argamassas, cerâmica, concreto, gesso, madeira, etc. O RCD no Brasil é composto por cerca de $63 \%$ de argamassa, $29 \%$ por concreto e blocos, $7 \%$ por outros resíduos e $1 \%$ de materiais orgânicos (SILVA, 2005 apud SANTOS, 2009).

Atualmente, a maioria dos países desenvolvidos ou que estão em desenvolvimento sofrem com a escassez de matéria-prima, como por exemplo, a brita e a areia. Para solucionar estes problemas de falta de insumos naturais, alguns países desenvolveram estudos para se utilizar o RCD para a produção de concreto. A Holanda, Bélgica e Dinamarca, são alguns desses países, que investiram em pesquisas para aproveitar o RCD e atualmente reciclam mais de $90 \%$ dos resíduos (CIOCCHI, 2003). A reciclagem no Brasil ainda é muito pequena se comparada a muitos países da Europa. O IBGE (2010) constatou que $72,45 \%$ dos municípios brasileiros apresentam o serviço de coleta de RCD, sendo que desses, apenas 9,7\% realizam alguma forma de processamento para realizar a reciclagem (ROMA; MOURA, 2011). Apesar do aumento crescente da reciclagem de RCD realizada por empresas privadas e pelo poder público, no Brasil a reciclagem ainda precisa ser mais estimulada. 
Os resíduos de concreto provenientes de centrais dosadoras, fábrica de pré-moldados, novas construções e demolições são significantes na composição total do RCD, aproximadamente $29 \%$ juntamente com blocos, por isso, é interessante o aproveitamento destes resíduos. Ciocchi (2003) afirma que a substituição de $25 \%$ dos agregados naturais por agregados reciclados provenientes de concreto não altera as propriedades mecânicas do concreto. $\mathrm{O}$ autor ainda destaca as vantagens econômicas de se obter um concreto reciclado com características semelhantes ao concreto convencional, a partir de recursos minerais com baixo custo.

O aproveitamento dos resíduos de concreto gerado por centrais dosadoras e fábricas de pré-moldados apresenta uma maior viabilidade do que os resíduos gerados por construções e demolições (BUTTLER, 2003). Os resíduos de concreto provenientes de RCD apresentam idades e propriedades de resistência não conhecidas e necessitam de uma separação, ou seja, apresentam impurezas e uma composição bastante heterogênea. A composição heterogênea do RCD influencia nas propriedades dos agregados reciclados, pois os diversos materiais componentes do RCD apresentam propriedades físicas diferentes. A utilização de resíduos de concreto provenientes de pré-moldados possibilita obter agregados reciclados com maior controle das propriedades do concreto reciclado produzido. De acordo com Prado (2006), as propriedades do concreto reciclado são influenciadas pelas características dos agregados reciclados utilizados, pelo teor de agregado natural substituído e pela quantidade de finos incorporados.

Os agregados reciclados de concreto apresentam diferenças significativas de suas propriedades físicas em relação aos agregados naturais. Segundo Leite (2001), a taxa de absorção é um fator que interfere na relação a/c das misturas, pois se ela não for considerada haverá uma redução da relação a/c e, conseqüentemente, a trabalhabilidade diminuirá. A massa específica dos agregados reciclados também se diferencia dos agregados naturais (BUTTLER, 2003). O fator determinante para o aumento da absorção de água e a redução da massa específica dos agregados reciclados de concreto, segundo os pesquisadores, foi a grande quantidade de argamassa aderida aos agregados reciclados. Esta argamassa aderida também influenciou na porosidade do agregado através da grande quantidade de vazios capilares, por isso, o índice de vazios dos agregados reciclados apresentou um valor alto em relação aos agregados naturais. A resistência e a idade do concreto que deu origem aos agregados reciclados são determinantes para os resultados da resistência à compressão do concreto reciclado, como também seus materiais componentes e a sua relação a/c (PRADO, 2006).

\section{PROGRAMA EXPERIMENTAL}

\subsection{Materiais}

O aglomerante utilizado neste trabalho foi o cimento CP V - ARI RS, cuja massa específica é de $3,07 \mathrm{~kg} / \mathrm{dm}^{3}$. A areia utilizada foi proveniente do Rio Jacuípe, em Feira de Santana. Utilizou-se uma brita de origem granítica, da Pedreira Laje, de Conceição do Jacuípe/BA. O agregado graúdo reciclado foi proveniente de resíduos de concreto de uma fábrica de pré-moldados localizada na cidade de Amélia Rodrigues/BA. Os resíduos de concreto coletados na fábrica passaram por um processo prévio de peneiramento, sendo utilizados todos os resíduos passantes na peneira $19 \mathrm{~mm}$ e retidos na peneira 4,8 $\mathrm{mm}$. Após serem peneirados, os resíduos de concreto passaram por uma triagem para a retirada de impurezas, como madeira, papel, barras de aço, plástico, entre 
outros. O peneiramento foi o único processo de beneficiamento utilizado. As características dos agregados estão apresentadas na Tabela 1.

Tabela 1: Resultados das propriedades físicas dos agregados

\begin{tabular}{|c|c|c|c|}
\hline Propriedades & AMN & AGN & AGR \\
\hline $\begin{array}{c}\text { Dimensão máxima } \\
\text { característica }\end{array}$ & $2,36 \mathrm{~mm}$ & $19 \mathrm{~mm}$ & $19 \mathrm{~mm}$ \\
\hline Módulo de Finura & 2,33 & 6,85 & 6,79 \\
\hline Massa Específica & $2,54 \mathrm{~kg} / \mathrm{dm}^{3}$ & $2,79 \mathrm{~kg} / \mathrm{dm}^{3}$ & $2,54 \mathrm{~kg} / \mathrm{dm}^{3}$ \\
\hline Massa unitária & $1,45 \mathrm{~kg} / \mathrm{dm}^{3}$ & $1,50 \mathrm{~kg} / \mathrm{dm}^{3}$ & $1,26 \mathrm{~kg} / \mathrm{dm}^{3}$ \\
\hline Absorção de água & $0,8 \%$ & $0,8 \%$ & $3,5 \%$ \\
\hline
\end{tabular}

\subsection{Produção e avaliação dos concretos}

A avaliação da influência da substituição do agregado natural pelo reciclado de concreto nas propriedades mecânicas é um dos objetivos deste trabalho e para isso as propriedades mecânicas do concreto como a resistência à compressão e a resistência à tração por compressão diametral foram escolhidas neste trabalho para medir as características de qualidade exigidas.

Alguns parâmetros foram definidos para a produção dos concretos utilizados neste trabalho, como descrito a seguir:

- Percentuais de substituição: foram definidos os percentuais de substituição do agregado graúdo natural pelo reciclado de 0 e $50 \%$, permitindo a comparação do concreto produzido com $50 \%$ de agregados graúdos reciclados (AGR), e o concreto de referência.

- Traços utilizados: os traços utilizados para as dosagens de concreto natural e concreto reciclado foram os mesmos, variando apenas a natureza do agregado graúdo. Portanto, foi utilizado agregado miúdo natural para todos os traços. Definiu-se dois traços com duas diferentes resistências características (fc) de $25 \mathrm{MPa}$ e $45 \mathrm{MPa}$. Para a mistura 45-REF (Tabela 2) utilizou-se o superplastificante Glenium 51 fabricado pela empresa Basf.

- Relação água/cimento (a/c): foram utilizadas duas relações a/c para dois diferentes traços: 0,69 para um concreto com fc igual a $25 \mathrm{MPa}$ e 0,40 para o concreto com fc igual a 45MPa.

Tabela 2 - Traços em massa e quantidades de materiais utilizados na produção dos

concretos

\begin{tabular}{|c|c|c|c|c|c|c|c|}
\hline \multirow{2}{*}{ Mistura } & \multirow{6}{*}{ Traço Unitário (em massa) } & \multicolumn{5}{|c|}{ Quantidade de materiais para 1 $\mathbf{~ m}^{\mathbf{3}}$ de } \\
\cline { 3 - 7 } & & C & AMN & AGN & AGR & Água & SP \\
\hline $25-$ REF & $1: 2,94: 2,97: 0,69(\%)$ & 311,1 & 914,7 & 924,0 & 0,0 & 214,7 & 0,0 \\
\hline $45-$ REF & $1: 1,14: 1,62: 0,40(0,08 \%$ SP) & 556,8 & 634,8 & 902,1 & 0,0 & 222,7 & 0,012 \\
\hline $25-$ RC & $1: 2,94: 2,97: 0,69(0 \%)$ & 311,1 & 914,7 & 462,0 & 385,8 & 214,7 & 0,0 \\
\hline $45-$ RC & $1: 1,14: 1,62: 0,40(0 \%)$ & 556,8 & 634,8 & 451,0 & 376,7 & 222,7 & 0,0 \\
\hline
\end{tabular}

$\mathrm{REF}$ - Concreto de referência; RC - Concreto reciclado; AMN - Agregado miúdo natural; AGN -

Agregado graúdo natural; AGR - Agregado graúdo reciclado; SP - Aditivo superplastificante 
Para os concretos reciclados foi realizada as compensações de volume em função das massas específicas dos agregados, bem como foi realizada a compensação parcial da taxa de absorção do agregado reciclado.

A mistura dos concretos foi estabelecida de acordo com método de produção de concreto proposto por Tam et al. (2005) e foi realizada em betoneira de eixo inclinado, com capacidade de 140 litros. A sequência de colocação dos materiais na betoneira e o tempo de mistura estão apresentados no fluxo da Figura 1.

Figura 1 - Fluxo da sequência de produção e tempo de mistura dos concretos convencionais e reciclados

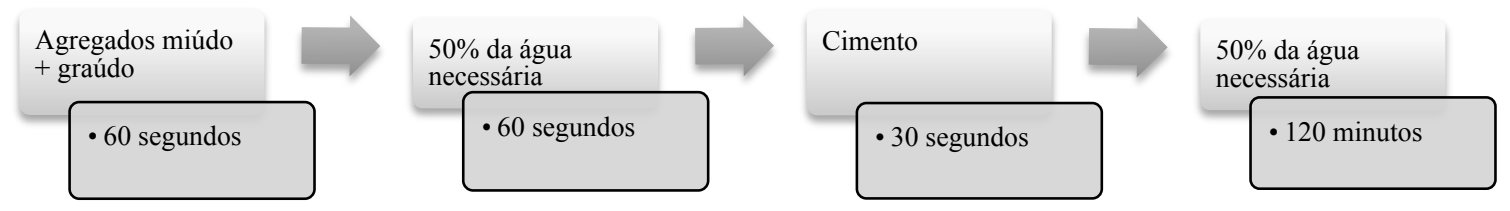

A moldagem e a cura dos corpos de prova foram realizadas de acordo com as especificações da NBR 5738 (ABNT, 2003). Foram utilizadas formas cilíndricas metálicas de $100 \mathrm{~mm}$ de diâmetro e $200 \mathrm{~mm}$ de altura para a moldagem dos corpos de prova. Nas primeiras 24 horas após a produção do concreto, a cura foi realizada ao ar, e depois de desmoldados até a data de realização dos ensaios, a cura foi feita por imersão em água com cal. O processo de adensamento adotado foi mecânico em apenas uma camada, de acordo com a norma citada anteriormente. Para o adensamento foi utilizado um vibrador de agulha de imersão.

Como mencionado anteriormente, os concretos foram avaliados quanto a determinação da resistência à compressão, de acordo com a prescrição da NBR 5739 (ABNT, 2007), e da resistência à tração por compressão diametral, de acordo com a prescrição da NBR 7222 (ABNT, 2011), aos 7 e 28 dias de idade do concreto. Foram moldados quatro de corpos de prova por idade, por ensaio a ser realizado.

\section{RESULTADOS E DISCUSSÃO}

\subsection{Resistência à compressão axial}

A resistência à compressão é considerada uma propriedade de grande importância no controle de qualidade do concreto. Alguns fatores são determinantes para se obter um bom desempenho na resistência do concreto, tais como, a relação a/c e as condições de cura, e especificamente para o concreto reciclado, o teor de agregados naturais substituídos por agregados reciclados é de grande relevância, pois afeta a porosidade da zona de transição entre a matriz e o agregado.

Os resultados obtidos dos ensaios de resistência à compressão dos concretos de referência e dos concretos reciclados estão apresentados na Tabela 3. 
Tabela 3 - Resultados de resistência à compressão do concreto de referência (REF) e do concreto reciclado $(\mathrm{RC})$

\begin{tabular}{|c|c|c|}
\hline \multirow{2}{*}{ Mistura } & $\mathbf{f}_{\mathbf{c}-\mathbf{7}}(\mathbf{M P a})$ & $\left.\mathbf{f}_{\mathbf{c}-\mathbf{2 8}} \mathbf{( M P a}\right)$ \\
\hline \multirow{2}{*}{25 REF } & 20,1 & 27,3 \\
\cline { 2 - 3 } & 18,8 & 25,8 \\
\cline { 2 - 3 } & 19,3 & 26,0 \\
\cline { 2 - 3 } & 18,9 & 25,9 \\
\hline \multirow{2}{*}{ Média (MPa) } & $\mathbf{1 9 , 3}$ & $\mathbf{2 6 , 3}$ \\
\hline \multirow{2}{*}{ Sd (MPa) } & $\mathbf{0 , 6}$ & $\mathbf{0 , 7}$ \\
\hline \multirow{2}{*}{ CV (\%) } & $\mathbf{3 , 0}$ & $\mathbf{2 , 6}$ \\
\hline \multirow{2}{*}{25 RC } & 19,0 & 22,8 \\
\cline { 2 - 3 } & 19,2 & 24,9 \\
\cline { 2 - 3 } & 19,8 & 25,0 \\
\hline \multirow{2}{*}{ Média (MPa) } & 19,8 & 25,5 \\
\hline Sd (MPa) & $\mathbf{1 9 , 4}$ & $\mathbf{2 4 , 5}$ \\
\hline CV (\%) & $\mathbf{0 , 4}$ & $\mathbf{1 , 2}$ \\
\hline
\end{tabular}

\begin{tabular}{|c|c|c|}
\hline \multirow{2}{*}{ Mistura } & $\left.\mathbf{f}_{\mathbf{c}-\mathbf{7}} \mathbf{( M P a}\right)$ & $\mathbf{f}_{\mathbf{c}-\mathbf{2 8}}(\mathbf{M P a})$ \\
\hline \multirow{3}{*}{$45 \mathrm{REF}$} & 33,6 & 40,2 \\
\cline { 2 - 3 } & 38,4 & 40,6 \\
\cline { 2 - 3 } & 36,5 & 42,9 \\
\cline { 2 - 3 } & 35,1 & 39,7 \\
\hline \multirow{2}{*}{ Média (MPa) } & $\mathbf{3 5 , 9}$ & $\mathbf{4 0 , 8}$ \\
\hline \multirow{2}{*}{ Sd (MPa) } & $\mathbf{2 , 1}$ & $\mathbf{1 , 4}$ \\
\hline \multirow{2}{*}{$\mathbf{C V}$ (\%) } & $\mathbf{5 , 7}$ & $\mathbf{3 , 6}$ \\
\hline \multirow{3}{*}{45 RC } & 38,0 & 43,2 \\
\cline { 2 - 3 } & 34,5 & 42,6 \\
\cline { 2 - 3 } & 34,5 & 42,2 \\
\cline { 2 - 3 } & 35,9 & 41,2 \\
\hline Média (MPa) & $\mathbf{3 5 , 7}$ & $\mathbf{4 2 , 3}$ \\
\hline Sd (MPa) & $\mathbf{1 , 7}$ & $\mathbf{0 , 8}$ \\
\hline CV (\%) & $\mathbf{4 , 6}$ & $\mathbf{1 , 9}$ \\
\hline
\end{tabular}

Os resultados de resistência relativa dos concretos estudados estão apresentados na Figura 2. A partir da análise desses resultados percebe-se que os concretos reciclados apresentaram resistências iguais ou maiores que as misturas de referência, exceto para a mistura 25RC aos 28 dias de idade que apresentou uma redução da resistência da ordem de $6 \%$ em relação à resistência do concreto de referência.

Figura 2 - Relação da resistência à compressão do concreto reciclado e a resistência à compressão do concreto de referência sobre as misturas, nas seguintes idades: a) 7 dias. b) 28 dias.

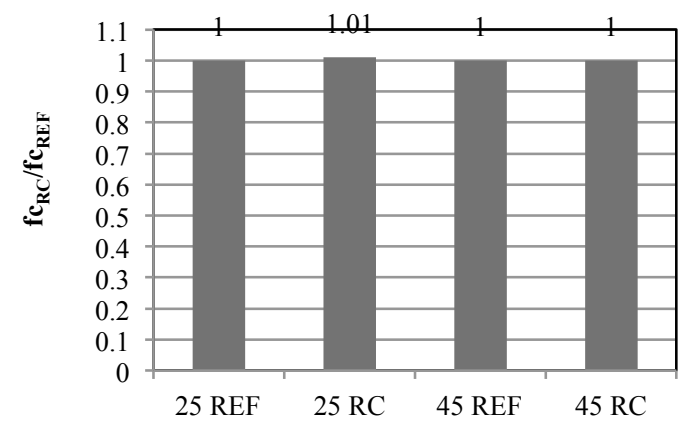

a)

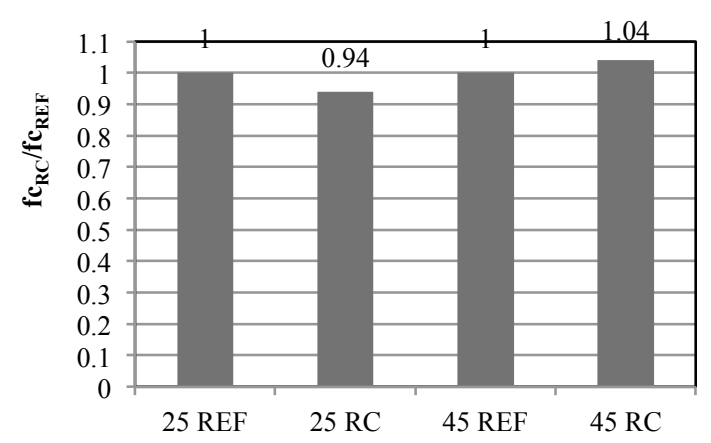

b)

Os resultados das misturas $25 \mathrm{RC}$, aos 7 dias, e a mistura $45 \mathrm{RC}$ aos 28 dias apresentaram uma resistência maior que o concreto de referência (Figura 2). O aumento da resistência dessas misturas, conforme constatado na literatura (BUTTLER, 2003; LEITE, 2001; PRADO, 2006) pode ser atribuído a grande quantidade de partículas de cimento não hidratadas, que podem ter sido hidratadas durante a nova concretagem e devido a 
migração da água do agregado para a pasta após o seu endurecimento, contribuindo para as reações de hidratação devido a um efeito de "cura úmida interna". Além disso, esse aumento pode estar relacionado com a melhoria da aderência pasta/agregado, com uma possível melhoria do empacotamento como também devido a não compensação da taxa de absorção, que pode ter reduzido um pouco a relação a/c na zona de transição.

Somado a esses fatores, a utilização do método de mistura proposto por Tam et al. (2005) pode ter sido umas das principais causas para o aumento da resistência à compressão do concreto reciclado. De acordo com os autores, a produção do concreto em duas etapas permite o preenchimento dos poros do agregados com uma fina camada de nata formada na primeira etapa da mistura, com isso torna a zona de transição mais forte e proporciona um aumento da resistência à compressão.

\subsection{Resistência à tração por compressão diametral}

Os resultados obtidos dos ensaios de resistência à tração por compressão diametral do concreto de referência e do concreto reciclado estão apresentados na Tabela 4.

Tabela 1 - Resultados de resistência à tração do concreto de referência (REF) e do concreto reciclado $(\mathrm{RC})$

\begin{tabular}{|c|c|c|c|c|c|}
\hline Mistura & $\mathbf{f}_{\mathrm{t}-7}(\mathrm{MPa})$ & $\mathbf{f}_{\mathrm{t}-28}(\mathrm{MPa})$ & Mistura & $\mathbf{f}_{\mathrm{t}-7}(\mathrm{MPa})$ & $f_{t-28}(M P a)$ \\
\hline \multirow{4}{*}{$25 \mathrm{REF}$} & 5,9 & 6,4 & \multirow{4}{*}{$45 \mathrm{REF}$} & 10,3 & 9,5 \\
\hline & $4,2^{*}$ & 7,2 & & $6,1^{*}$ & 9,9 \\
\hline & 5,0 & $5,7^{*}$ & & 10,9 & 11,1 \\
\hline & 5,9 & 7,4 & & $7,3^{*}$ & $8,2 *$ \\
\hline Média (MPa) & 5,6 & 7,0 & Média (MPa) & 10,6 & 10,2 \\
\hline Sd (MPa) & 0,5 & 0,5 & Sd (MPa) & 0,4 & 0,8 \\
\hline CV (\%) & 9,0 & 7,5 & CV (\%) & 4,2 & 8,2 \\
\hline \multirow{4}{*}{$25 \mathrm{RC}$} & $4,8^{*}$ & $5,9^{*}$ & \multirow{4}{*}{$45 \mathrm{RC}$} & 10,2 & 12,0 \\
\hline & 6,3 & 7,8 & & $6,0^{*}$ & $7,8^{*}$ \\
\hline & 5,1 & 8,2 & & 11,5 & 11,4 \\
\hline & $4,5^{*}$ & 8,7 & & $5,9 *$ & 10,2 \\
\hline Média (MPa) & 5,7 & 8,2 & Média (MPa) & 10,9 & 11,2 \\
\hline Sd (MPa) & 0,8 & 0,4 & Sd (MPa) & 0,9 & 0,9 \\
\hline CV (\%) & 14,9 & 5,3 & CV (\%) & 8,6 & 8,1 \\
\hline
\end{tabular}

*Resultado excluído do cálculo da média e do desvio padrão $(\mathrm{Sd})$.

A partir dos resultados da Tabela 4 percebe-se que as misturas dos concretos com o maior consumo de cimento, ou seja, as misturas 45RC e 45REF apresentaram o menor ganho resistência com o tempo, comparando as resistências aos 7 e 28 dias de idade. A resistência à tração do concreto reciclado dos dois traços utilizados foram maiores que o concreto convencional, conforme se observa na Figura 3. Portanto, a utilização do agregado graúdo reciclado de concreto foi interessante para obtenção de uma maior resistência à tração. 
Figura 3 - Relação da resistência à compressão do concreto reciclado e a resistência à tração por compressão diametral do concreto de referência sobre as misturas, nas seguintes idades: a) 7 dias; b) 28 dias

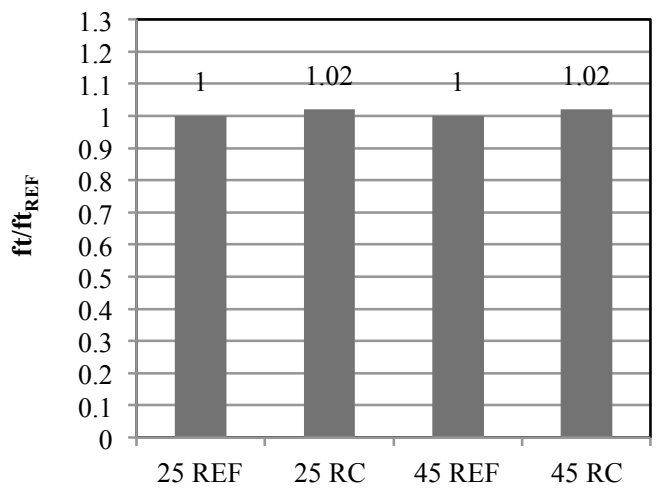

a)

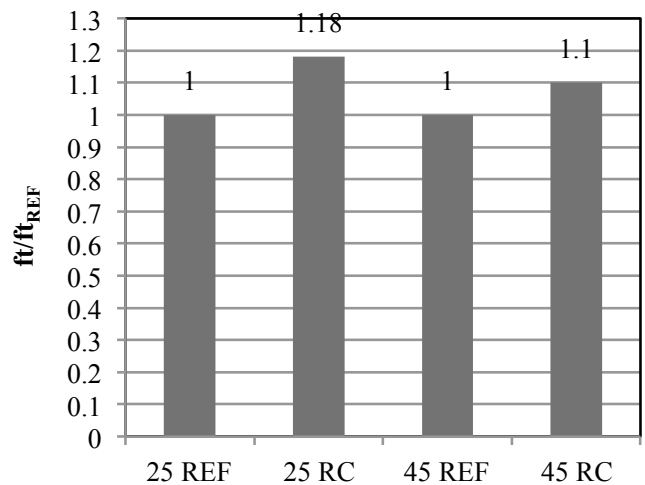

b)

De acordo com Neville (1997), a resistência à compressão e à resistência à tração são estritamente relacionadas, porém não são proporcionais diretamente. As resistências à compressão e à tração aumentam, mas a uma razão decrescente. A razão entre a resistência à tração direta/compressão é de 10 a $11 \%$ para concreto de baixa resistência, 8 a 9\% para concreto de média resistência e da ordem de $7 \%$ para concreto de alta resistência (METHA; MONTEIRO, 1994). A Tabela 5 apresenta as relações entre a resistência à tração e a resistência à compressão das misturas avaliadas.

Tabela 2: Relação entre a resistência à tração e a resistência à compressão das misturas dos concretos estudados

\begin{tabular}{|c|c|c|c|c|c|c|}
\hline & & & \multicolumn{4}{|c|}{ Mistura } \\
\hline & & & $25 \mathrm{REF}$ & $25 \mathrm{RC}$ & $45 \mathrm{REF}$ & $45 \mathrm{RC}$ \\
\hline \multirow{6}{*}{$\begin{array}{l}\frac{\pi}{\pi} \\
\text { U }\end{array}$} & \multirow{3}{*}{7 dias } & fc $(\mathrm{MPa})$ & 19,3 & 19,5 & 35,9 & 35,7 \\
\hline & & $\mathrm{ft}(\mathrm{MPa})$ & 5,6 & 5,7 & 10,6 & 10,9 \\
\hline & & $\mathrm{ft} / \mathrm{fc}(\%)$ & 29,1 & 29,3 & 29,5 & 30,4 \\
\hline & \multirow{3}{*}{28 dias } & $\mathrm{fc}(\mathrm{MPa})$ & 26,3 & 24,6 & 40,9 & 42,3 \\
\hline & & $\mathrm{ft}(\mathrm{MPa})$ & 7,0 & 8,2 & 10,2 & 11,2 \\
\hline & & $\mathrm{ft} / \mathrm{fc}(\%)$ & 26,7 & 33,5 & 24,9 & 26,5 \\
\hline
\end{tabular}

Observa-se que as relações $\mathrm{ft} / \mathrm{fc}$ obtidas foram consideravelmente superiores em relação ao que ocorre normalmente para qualquer dos concretos avaliados. Aos 28 dias de idade os concretos reciclados apresentaram maiores relações entre tração e compressão, como era de se esperar, pela melhoria na zona de transição. Também se pode perceber que no concreto convencional assim como no concreto reciclado ocorre uma redução da relação $\mathrm{ft} / \mathrm{fc}$ à medida que a resistência à compressão do concreto aumenta.

As relações da resistência à tração e a resistência à compressão desta pesquisa apresentados na Tabela 5 foram maiores que o valor comumente apresentado na bibliografia, que é de aproximadamente 10\%. Alguns motivos podem explicar este aumento: 
- a melhor aderência da pasta ao agregado contribuiu para aumentar mais a resistência à tração que a compressão;

- produção do concreto com uma zona de transição menos porosa;

- melhor hidratação dos grãos de cimento na zona de transição;

- boa qualidade do concreto e dos agregados.

Leite (2001) também analisou a relação ft/fc e obteve relações maiores que $10 \%$ para todas as relações a/c avaliadas em seu estudo. Na sua pesquisa, observou-se que a utilização de agregados reciclados de maior dimensão na produção de concretos melhora os resultados da resistência à tração. Portanto, a utilização de agregados com uma dimensão máxima de $19 \mathrm{~mm}$ neste trabalho, pode ter favorecido ao aumento da resistência à tração. Assim como nesta pesquisa, a autora também destaca a ocorrência da diminuição da relação ft/fc à medida que a resistência à compressão do concreto aumenta no concreto reciclado.

\section{CONSIDERAÇÕES FINAIS}

A preocupação com o meio ambiente está proporcionando o aparecimento de grandes inovações tecnológicas para reduzir os impactos gerados a partir dos vários processos produtivos. A reciclagem dos resíduos tem se mostrado uma alternativa para a redução da poluição do meio ambiente, além de reduzir o consumo de materiais extraídos da natureza. Para realizar o reaproveitamento é necessário que seja ampliado cada vez mais o conhecimento sobre o comportamento dos resíduos, como é o caso do RCD na produção de novos concretos.

Os resultados desse estudo apontaram que tanto a resistência à compressão quanto a resistência à tração por compressão diametral do concreto produzido com a substituição de $50 \%$ dos agregados naturais graúdos por agregados reciclados de concreto não foram afetadas. De um modo geral, os resultados de resistência à compressão do concreto com agregado reciclado de concreto apresentaram valores iguais ou superiores ao concreto de referência e as resistências à tração apresentaram valores superiores aos do concreto convencional. Acredita-se que a rugosidade do agregado reciclado de concreto e o método de mistura utilizado foram determinantes para melhoria do desempenho mecânico dos concretos reciclados.

Além das vantagens técnicas, pode-se dizer que a produção de concreto reciclado usando agregados reciclados de concreto também apresenta vantagens econômicas, uma vez que o uso de agregados extraídos de fontes naturais podem ser reduzidos e o resíduo utilizado terá uma destinação mais nobre que o simples aterramento.

\section{AGRADECIMENTOS}

Os autores agradecem à CAPES e ao CNPq pelo apoio recebido. 


\section{REFERÊNCIAS}

ASSOCIAÇÃO BRASILEIRA DE NORMAS TÉCNICAS. NBR 5738: Concreto Procedimento para moldagem e cura de corpos-de-prova. Rio de Janeiro, 2003. Janeiro, 2007.

NBR 5739: Concreto - Ensaios de compressão de corpos-de-prova cilíndricos. Rio de

NBR 7222: Concreto e argamassa - Determinação da resistência à tração por compressão diametral de corpos de prova cilíndricos. Rio de Janeiro, 2011.

BUTTLER, Alexandre Marques. Concreto com agregados graúdos reciclados de concreto : influência da idade de reciclagem nas propriedades dos agregados e concretos reciclados. São Carlos: USP, 2003. Dissertação (Mestrado em Engenharia Civil), Escola de Engenharia de São Carlos da Universidade de São Paulo, Universidade de São Paulo, 2003.

CIOCCHI, Luis. Reciclagem de Concreto. Piniweb, 2003. Disponível em:

http://www.piniweb.com.br/construcao/noticias/reciclagem-de-concreto-80112-1.asp Acesso em 13 nov. 2011.

LEITE, Mônica Batista. Avaliação de propriedades mecânicas de concretos produzidos com agregados reciclados de resíduos de construção e demolição. Porto Alegre: UFRGS, 2001. Tese (Doutorado em Engenharia Civil), Programa de Pós Graduação em Engenharia Civil, Universidade Federal do Rio Grande do Sul, 2001.

MEHTA, Povindar K.; Monteiro, Paulo J. Melaragno. Concreto: estrutura, propriedades e materiais. São Paulo: Pini, 1994.

NEVILle, Adam M. Propriedades do Concreto. 2 ed. São Paulo: Pini, 1997.

PINTO, T. P.; GONZÁLES, J. L. R. (Coord.). Manejo e gestão dos resíduos da construção civil. Volume 1 - Manual de orientação: como implementar um sistema de manejo e gestão nos municípios. Brasília: CAIXA,2005.194p. ,PINI.

PRADO, D. M. Propriedades físicas e mecânicas de blocos estruturais produzidos com agregados reciclados de concreto. 2006. 140p. Dissertação de Mestrado, Universidade de São Paulo. Escola de Engenharia de Estruturas, São Carlos.

ROMA, J. C.; MOURA, A. A. Resíduos da Construção Civil. 2011. 46 p. Caderno de Diagnóstico. Instituto de Pesquisa Econômica Aplicada.

SANTOS, A. L. Diagnóstico ambiental da gestão e destinação dos resíduos de construção e demolição (RCC): análise das construtoras Associadas ao SINDUSCON/RN e empresas coletoras atuantes no município de Parnamirim - RN. 2009. 107p. Dissertação (mestrado). Universidade Federal do Rio Grande do Norte. 2009.

TAM, V.W.Y.;GAO, X.F.;TAM, C.M. Microestructural analysis of recycled aggregate concrete produced from two-stage mixing approach. Cement and Concrete Research, v.35, n.6,p; 1195-1203,2005. 